\title{
Dynamic contrast-enhanced magnetic resonance imaging and pharmacokinetic models in prostate cancer
}

\author{
Tobias Franiel • Bernd Hamm • Hedvig Hricak
}

Received: 19 September 2010 /Revised: 16 November 2010 /Accepted: 25 November 2010 /Published online: 24 December 2010

(C) European Society of Radiology 2010

\begin{abstract}
Dynamic contrast-enhanced MRI enables noninvasive analysis of prostate vascularization as well as tumour angiogenesis and capillary permeability characteristics in prostate cancers. Pharmacokinetic models summarizing the complex information provided by signal intensity-time curves for a few quantitative pharmacokinetic parameters are increasingly being used in the routine clinical setting. This review consists of two parts. The first part discusses the advantages and disadvantages of the MR pulse sequences that can be used for performing DCE-MRI and also of the most widely used pharmacokinetic parameters and models and the parameters they describe. The second part outlines the range of current and potential future clinical applications of DCE-MRI and pharmacokinetic parametric maps in patients with prostate cancer, with reference to the current scientific literature on the topic. The potential clinical applications of DCE-MRI for prostate cancer include detection, localization, and staging, differentiation of recurrent cancer and estimation of the patient's prognosis, as well as monitoring of treatment response.
\end{abstract}

T. Franiel $\cdot$ H. Hricak

Department of Radiology,

Memorial Sloan-Kettering Cancer Center,

New York, NY 10021, USA

\section{B. Hamm}

Department of Radiology, Charité - Universitätsmedizin Berlin,

Charitéplatz 1,

10117 Berlin, Germany

\section{T. Franiel $(\bowtie)$}

Institut für Radiologie, Charité, Universitätsmedizin Berlin,

Charitéplatz 1,

10117 Berlin, Germany

e-mail: tobias.franiel@charite.de
Keywords Prostate $\cdot$ DCE-MRI $\cdot$ Pharmacokinetic parameters $\cdot$ Blood flow $\cdot$ Permeability

\section{Introduction}

Prostate cancer is the most common malignant tumour and the second leading cause of cancer death in men [1]. Diagnostic work-up focuses on early detection, determination of biological aggressiveness, localization within the prostate, staging, evaluation of the outcome of treatment, and detection of recurrent cancer [2]. Imaging in general, and magnetic resonance imaging (MRI) in particular, is assuming a more important role in addressing these diagnostic tasks. T2-weighted (w) MR images typically show prostate cancer as a focal lesion of low signal intensity [3]. This low T2 signal intensity, however, is not specific to prostate cancer, other abnormal conditions of the prostate that may have the same or similar signal intensity include prostatitis, hemorrhage, fibrosis, glandular atrophy, and post-therapeutic changes [4-6]. To improve the specificity of MRI, the conventional $\mathrm{T} 2 \mathrm{w}$ and $\mathrm{T} 1 \mathrm{w}$ sequences are increasingly being supplemented by functional MRI techniques such as ${ }^{1} \mathrm{H}$ magnetic resonance spectroscopy $\left({ }^{1} \mathrm{H}-\mathrm{MRS}\right)$, diffusion-weighted imaging (DWI), and dynamic contrast-enhanced MRI (DCE-MRI) [7]. Initial studies of contrast-enhanced MRI suggest that after administration of a low-molecular-weight contrast agent, only dynamic and not static $\mathrm{T} 1 \mathrm{w}$ sequences contribute diagnostic information additional to that obtained from unenhanced T2w imaging alone [8-10].

With DCE-MRI, prostate cancer shows earlier and stronger enhancement than surrounding normal prostate tissue [10]. This enhancement pattern is based on tumour angiogenesis, an important prerequisite for further growth 
once a cancer has reached a size of $3 \mathrm{~mm}[11,12]$. The newly formed tumour vessels have higher permeability, and their architecture is heterogeneous $[13,14]$. Immunohistochemical determination of mean vessel density (MVD) is a good method for assessing tumour angiogenesis. This method provides a count of the number of vessels per area, which is significantly higher in prostate cancer than in normal prostate tissue $[15,16]$. Published data on the significance of MVD as a predictor of recurrence, presence of metastasis, or disease-specific survival are contradictory [17-21]. More recent results suggest that a poorer prognosis correlates not only with a higher MVD but also with increasing irregularity and decreasing size of the new vessels $[20,22]$. These histological observations are the rationale for the high hopes set on the potential of DCEMRI for noninvasively and dynamically assessing microvascularization and neoangiogenesis in prostate cancer.

The multifarious and complex information provided by signal intensity-time curves generated on the basis of DCEMRI datasets can be described by phenomenological parameters or extracted and summarized in a few quantitative pharmacokinetic parameters. The latter is accomplished by applying pharmacokinetic models that describe tissue vascularization and blood flow in the form of mathematical equations. The parametric maps generated by means of these models facilitate interpretation of the DCE-MRI data, and it is therefore expected that the use of pharmacokinetic models will increase in the routine clinical setting. The first part of this review discusses the advantages and disadvantages of MR pulse sequences for performing DCE-MRI and of the most widely used pharmacokinetic models and parameters. The second part outlines the range of current and potential future clinical applications of DCE-MRI and pharmacokinetic parameters in prostate cancer. For ease of reading, we deliberately abstained from using mathematical formulas in describing the technique. The interested reader is referred to the more detailed literature quoted in the respective passages of this review. Moreover, we only discuss the widely used unspecific low-molecular-weight contrast agents. Following intravenous administration, these agents distribute in the intravascular and interstitial spaces throughout the body but do not cross the normal cerebral blood-brain barrier.

\section{Technique of DCE-MRI and computation of parametric pharmacokinetic maps}

This section is divided into two parts, following the procedure of generating pharmacokinetic parameter maps. The first part describes how signal intensities (SI) are measured using DCEMRI and how these are converted into contrast medium (CM) concentrations $([\mathrm{CM}])$. The second part deals with the arterial input function and the pharmacokinetic models used. These models can in principle be applied regardless of whether [CM] is measured invasively or whether it is derived from the SI of noninvasively acquired MRI data. A protocol for an MRI examination of the prostate combining conventional MR pulse sequences with a DCE-MRI sequence is proposed in Table 1.

\section{DCE-MRI}

A DCE-MRI sequence is acquired at multiple consecutive time-points for measuring tissue SI before, during and after $\mathrm{CM}$ administration. T1w SI-time curves were described in the past using phenomenological parameters such as start of enhancement, time to peak, peak enhancement, and washout [10]. Start of enhancement is the point in time at which the first tissue enhancement is noted after CM administration, time to peak is the time interval until the SI plateau is reached, peak enhancement is the maximum SI, and washout is the percentage of SI loss after the peak has been reached [23]. To calculate pharmacokinetic parameters from the SI-time curves, the SI must be converted into [CM]. How the SI are converted into $[\mathrm{CM}]$ is determined by the MR pulse sequence used. When an ultrafast saturation recovery (SR) spoiled gradient echo (GRE) sequence with a short time to repeat (TR), short time to echo (TE), and small flip angle is used, the $2^{*}$ susceptibility effect is negligible, as the TE is much shorter than the $\mathrm{T} 2 *$ time [24]. Using these parameters and a recovery time of $\leq 150 \mathrm{~ms}$, this technique provides a good approximation of the relative SI change after CM administration, which is linear to the local $[\mathrm{CM}]$ in the tissue voxel over a large dose range [24]. If the SR prepulse is replaced with an inversion recovery (IR) prepulse, all of the longitudinal magnetization is available for measuring SI, which is why an IR pulse sequence is more sensitive to changes in relaxivity; however, the image acquisition time is also longer. Once again, a linear relationship can be expected between SI and $[\mathrm{CM}]$ for the low $[\mathrm{CM}]$ range of $\leq 4 \mathrm{mmol} / \mathrm{l}$; the concentration is likely to be within this range, unless images are acquired in the early distribution phase [25]. However, if the MR sequence also serves to adequately quantify the early distribution phase, especially the first pass of the $\mathrm{CM}$, then one must consider the $\mathrm{T} 2 *$ effects as well; these effects are proportional to $[\mathrm{CM}]$ and reduce the T1 signal $[25,26]$. To capture these effects, a dual-echo GRE sequence is used, which acquires $\mathrm{T} 1 \mathrm{w}$ and $\mathrm{T} 2 * \mathrm{~W}$ images simultaneously [25-27]. The dual-echo sequence thus allows sensitive measurement of the SI of low tissue $[\mathrm{CM}]$ along with the sensitive measurement of the SI of high $[\mathrm{CM}]$ for extracting the individual AIF.

Ultrafast spoiled dual- or single-pulse GRE sequences without a prepulse are faster than SR or IR GRE sequences, 
Table 1 Suggested protocol for MRI of the prostate using the combined endorectal/body phased-array coil, including two halfFourier rapid acquisition with relaxation enhancement (RARE) sequences for planning $(1 .+2$.$) , conventional \mathrm{T} 1 \mathrm{w}$ and $\mathrm{T} 2 \mathrm{w}$ turbo spin echo (TSE) sequences $(3 .+4 .+5$.$) , a sequence for lymph node$ evaluation from the aortic bifurcation to the true pelvis (6.), a volumeinterpolated gradient echo (GRE) sequence for calculating R1 relaxivity prior to contrast medium administration (7.), and a dynamic spoiled axial T1w GRE sequence with 75 acquisitions (each 3.9 s) (8.)

\begin{tabular}{|c|c|c|c|c|c|c|c|c|c|c|c|}
\hline & $\begin{array}{l}\text { Time to } \\
\text { repeat } \\
{[\mathrm{ms}]}\end{array}$ & $\begin{array}{l}\text { Time } \\
\text { to } \\
\text { echo } \\
{[\mathrm{ms}]}\end{array}$ & $\begin{array}{l}\text { Flip angle } \\
{\left[^{\circ}\right]}\end{array}$ & $\begin{array}{l}\text { Echo } \\
\text { train } \\
\text { length }\end{array}$ & $\begin{array}{l}\text { Number } \\
\text { of } \\
\text { averages }\end{array}$ & $\begin{array}{l}\text { Field } \\
\text { of } \\
\text { view } \\
{[\mathrm{mm}]}\end{array}$ & Matrix & $\begin{array}{l}\text { Slice } \\
\text { thickness } \\
{[\mathrm{mm}]}\end{array}$ & $\begin{array}{l}\text { Interslice } \\
\text { gap } \\
{[\mathrm{mm}]}\end{array}$ & $\begin{array}{l}\text { Parallel } \\
\text { imaging } \\
\text { technique }\end{array}$ & $\begin{array}{l}\text { Imaging } \\
\text { time } \\
{[\mathrm{min}]}\end{array}$ \\
\hline $\begin{array}{l}\text { 1. Sagittal half- } \\
\text { Fourier } \\
\text { RARE }\end{array}$ & 800 & 80 & 180 & - & 1 & $\begin{array}{r}320 \times \\
288\end{array}$ & $\begin{array}{r}320 \times \\
288\end{array}$ & 7 & 2.1 & mSENSE & $\begin{array}{l}\text { Ca. } \\
0.25\end{array}$ \\
\hline $\begin{array}{l}\text { 2. Axial half- } \\
\text { Fourier } \\
\text { RARE }\end{array}$ & 800 & 80 & 180 & - & 1 & $\begin{array}{r}370 \times \\
333\end{array}$ & $\begin{array}{r}320 \times \\
288\end{array}$ & 7 & 2.1 & mSENSE & $\begin{array}{l}\text { Ca. } \\
0.25\end{array}$ \\
\hline $\begin{array}{l}\text { 3. Axial T2w } \\
\text { TSE }\end{array}$ & 4850 & 85 & 150 & 15 & 2 & $\begin{array}{r}160 \times \\
160\end{array}$ & $\begin{array}{r}256 \times \\
256\end{array}$ & 3.0 & 0.6 & - & Ca. 6 \\
\hline $\begin{array}{l}\text { 4. Coronal } \\
\text { T2w TSE }\end{array}$ & 4000 & 95 & 150 & 13 & 2 & $\begin{array}{r}200 \times \\
200\end{array}$ & $\begin{array}{r}256 \times \\
256\end{array}$ & 3.0 & 0.6 & - & Ca. 6 \\
\hline $\begin{array}{l}\text { 5. Axial T1w } \\
\text { TSE }\end{array}$ & 691 & 12 & 150 & 3 & 1 & $\begin{array}{r}160 \times \\
160\end{array}$ & $\begin{array}{r}256 \times \\
256\end{array}$ & 3.0 & 0.6 & - & Ca. 3 \\
\hline $\begin{array}{l}\text { 6. Axial T1/ } \\
\text { PDw TSE }\end{array}$ & 1200 & 13 & 150 & 3 & 2 & $\begin{array}{r}320 \times \\
240\end{array}$ & $\begin{array}{r}512 \times \\
512\end{array}$ & 7 & 1.4 & - & Ca. 5 \\
\hline $\begin{array}{l}\text { 7. Volume- In- } \\
\text { terpolated } \\
\text { GRE } \\
\text { Sequenz (4x) }\end{array}$ & 4.57 & 1.63 & $2,5,10,15$ & - & 1 & $\begin{array}{r}260 \times \\
260\end{array}$ & $\begin{array}{r}256 \times \\
256\end{array}$ & 3.6 & 0 & GRAPPA & 2.7 \\
\hline $\begin{array}{l}\text { 8. Dynamic } \\
\text { axial spoiled } \\
\text { T1w GE }\end{array}$ & 5.19 & 2.02 & 15 & - & 1 & $\begin{array}{r}260 \times \\
260\end{array}$ & $\begin{array}{r}256 \times \\
256\end{array}$ & 3.6 & 0 & GRAPPA & $\begin{array}{l}4.9 \\
(75 \times \\
3.9 \mathrm{~s})\end{array}$ \\
\hline
\end{tabular}

but this comes at the cost of a poorer signal-to-noise ratio. When an ultrafast spoiled T1w 3D single GRE sequence without a prepulse is used, the relationship between SI and [CM] is complex and typically nonlinear, and it is not possible to directly derive [CM] from SI. However, in this case, the desired information can be extracted via an additional step: this is the calculation of the relative change in relaxivity, $\Delta \mathrm{R} 1$, of the tissue following $\mathrm{CM}$ administration. This parameter is in a linear and directly proportional relationship to [CM] based on the relaxivity of the $\mathrm{CM}$ used [27]. Tissue relaxivity $\mathrm{R} 1$ can be calculated, at any point in time, from the measured signal intensity, the parameters of the pulse sequence used, and the relaxivity, $\mathrm{R} 1_{0}$, before $\mathrm{CM}$ administration $[28,29]$. Relaxivity, $\mathrm{R} 1_{0}$, of the entire tissue in turn can be calculated from the SI of at least two acquisitions with different flip angles and the TR of the pulse sequence used [30]. Determination of tissue SI from images acquired with 3 or 4 different flip angles minimizes the effects of noise [30].

\section{Arterial input function}

Pharmacokinetic model calculations require the so-called arterial input function (AIF), which is the intra-arterial [CM] over time. There are basically two approaches: to use a standardized AIF or to calculate an individual AIF for each patient. Determination of an individual AIF provides an optimal basis for the correct extraction of the desired pharmacokinetic parameters, as it accounts for interindividual variations in cardiac output, renal function, and hematocrit levels, which influence the individual [CM] entering the organ $[31,32]$. An individual AIF is calculated from the measured MR SI in a large artery, ideally an artery supplying the organ of interest. The measured MR SI are converted into [CM] by fitting an analytical concentrationtime curve to the SI-time curves measured in the central artery, which is subject to the dependencies already described in the DCE-MRI section above. As a result of the highly concentrated CM bolus, especially during first pass, the measured T1w SI must be corrected for the signallowering T2* effect $[25,26]$. Temporal resolution should not exceed $2 \mathrm{~s}$ in order to ensure adequate tracking of the arrival and passage of the contrast medium bolus [33]. To our knowledge, with the MR techniques currently in clinical use, these prerequisites are only met when a single slice is imaged. A single slice is sufficient to determine the AIF, but evaluation of the prostate in a single slice severely degrades the clinical usefulness of the method. For the time being, it appears justified to use a standardized AIF to overcome this limitation in the clinical setting and acquire 
several slices of the prostate. In routine clinical examinations, interindividual variations in blood volume are taken into account by adjusting the contrast medium dose to the patient's weight [34]. Creatinine levels and the calculated glomerular filtration rate (e.g., using the Cockcroft-Gault formula) should be determined routinely to rule out excessively reduced renal elimination. One of the first standardized AIFs was determined invasively from blood samples in 20 healthy men following administration of GdDTPA; the first blood sample for measurement was obtained 1 min after CM administration [35]. This study demonstrated a biexponential decrease in $[\mathrm{CM}]$ in the body with a half-life of $12 \mathrm{~min}$ for the distribution phase and a half-life of $1 \mathrm{~h}$ and $35 \mathrm{~min}$ for the elimination phase [35]. This AIF has a poor temporal resolution and is of limited value as most current protocols use bolus infusion of contrast medium. Invasive measurement of blood [CM] every $2 \mathrm{~s}$ in healthy volunteers clearly revealed a first-pass peak, a recirculation peak, and washout of the concentration in blood [36]. A similar concentration-time course was identified in a study determining a standardized AIF in patients with tumours [37]. It is therefore recommended to preferably use the standardized AIF reflecting both the firstpass peak and the recirculation peak.

\section{Pharmacokinetic models}

A widely used pharmacokinetic model for describing contrast medium kinetics in biological tissues is an open 2-compartment model with blood plasma as the central compartment and the interstitial space as the peripheral compartment $[38,39]$. In this model, it is assumed that the $\mathrm{CM}$ enters the central compartment with zero-order kinetics, while its elimination from it is characterized by the first-order elimination rate constant. Exchange between the two compartments is by passive, first-order diffusion in both directions, with a specific exchange constant for each direction. Assuming immediate distribution of the $\mathrm{CM}$ in plasma and neglecting the intravascular portion of the $\mathrm{CM}$, the time course of interstitial [CM], and hence of SI, is solely determined by the exchange constants between the central and the peripheral compartment [40]. When the relaxivity $\mathrm{R} 1_{0}$ of the target tissue before $\mathrm{CM}$ administration, the increase in relaxivity, $\mathrm{R} 1$, relative to $[\mathrm{CM}]$ in the tissue, and the blood plasma volume are known, the contribution of the extracellular, extravascular volume to the total volume of a voxel can be calculated [41, 42]. Following the recommendations of a consensus paper, the following terminology will be used here: EES for the extracellular, extravascular volume, transfer constant $\left(\mathrm{K}^{\text {trans }}\right)$ for the exchange constant between blood plasma and EES, and rate constant $\left(\mathrm{k}_{\mathrm{ep}}\right)$ for the exchange constant between EES and blood plasma [33] (Fig. 1). The open 2- compartment model assumes immediate distribution of $\mathrm{CM}$ in both arterial and venous blood plasma [39, 41, 42]. If, in addition, CM blood flow in the capillaries is large enough to compensate for diffusion into the EES, a permeabilitylimited model can be used [33]. In this case, diffusion of the CM from the blood plasma into the EES is only dependent on permeability, i.e., the permeability surface product of the vessels. However, capillaries are very fragile, especially in a tumour, making them highly permeable to a lowmolecular-weight contrast agent, and, in most cases, diffusion into the interstitial space probably cannot be compensated for by CM blood flow [43, 44]. In these cases, the exchange parameters are a function of permeability and $\mathrm{CM}$ blood flow. However, the model does not specify these dependencies and the magnitude of the two parameters, which is a serious limitation, given the role of these two parameters in describing $\mathrm{CM}$ kinetics. More complex models enable absolute quantification of blood flow, permeability, and blood volume. These models cover the whole range of possibilities from permeability-limited states to blood flow-limited states [33, 45, 46]. The mixed blood flow- and permeability-limited model describes changes in $[\mathrm{CM}]$ in the EES as a function of blood flow, the $[\mathrm{CM}]$ in blood plasma, and of the extraction coefficient, which is a measure of the amount of CM extravasating into the interstitial volume with each passage through the capillary bed of the prostate $[33,45]$. It has been shown that models comprising more than one tumour EES are superior for describing contrast medium kinetics when a tumour is present [38]. This observation may be attributable to the predominantly heterogeneous vascular architecture of prostate cancers, resulting in contrast medium diffusion pathways of variable length in the interstitial volume [17]. Therefore, an open sequential 3-compartment model has been proposed and used for the prostate and prostate cancer. This model comprises blood plasma as one compartment and two EES compartments [25, 47]. Using this model, quantification of blood flow in a tissue voxel is based on the so-called indicator dilution method and the calculated AIF [48]. Here, the extraction coefficient is a function of permeability, vessel surface area, and blood flow [33, 45]. Assuming that blood vessels are cylindrical, the vessel surface can be calculated from the blood volume and the radius; permeability and blood flow can thus be calculated as independent parameters [47]. A diagram of how pharmacokinetic parameters are calculated from SI measured with DCE-MRI and using a sequential 3compartment model is presented in Fig. 2.

In summary, it is theoretically possible to have a temporal resolution above $2 \mathrm{~s}$, if exchange constants are calculated using a standardized AIF. In this case, pharmacokinetic model calculations only require measurement of SI for rather low $[\mathrm{CM}]$ in tissue. At these concentrations, the $\mathrm{T} 2 *$ effect on the 


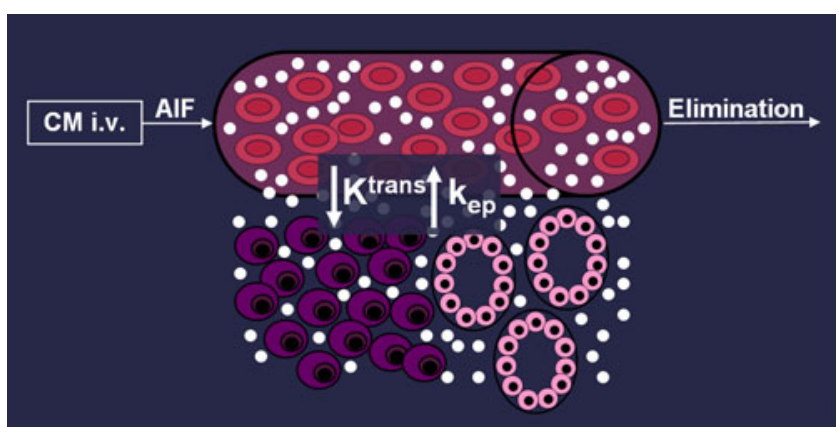

Fig. 1 Diagram of the open 2-compartment model with the two pharmacokinetic parameters, $\mathrm{K}^{\text {trans }}$ and $\mathrm{k}_{\mathrm{ep}}$. An unspecific lowmolecular-weight contrast agent (white dots) distributes exclusively in the intravascular blood plasma and in the extravascular extracellular space (EES). The transfer constant, $\mathrm{K}^{\text {trans }}$, describes the diffusion of the intravascular contrast medium $(\mathrm{CM})$ into the EES. When distribution in the body and renal elimination cause the contrast medium concentration $[\mathrm{CM}]$ in the plasma to drop below that in the EES, CM from the EES diffuses back into the plasma, which is described by the rate constant, $\mathrm{k}_{\mathrm{ep}}$

T1w signal is negligible, and the use of an ultrafast $\mathrm{T} 1 \mathrm{w}$ spoiled single-GRE sequence is justified. Conversely, a dualecho sequence for the simultaneous acquisition of $\mathrm{T} 1 \mathrm{w}$ and $\mathrm{T} 2 * \mathrm{w}$ images with a high temporal resolution of $2 \mathrm{~s}$ or less is required [33] to calculate an individual AIF. This has been shown to improve the accuracy of pharmacokinetic model calculations [31, 32] and is a prerequisite for the accurate calculation of blood flow and blood volume.

\section{Current and future clinical applications}

The studies quoted in this section analyzed DCE-MRI datasets using either phenomenological parameters or parameters derived with a pharmacokinetic model. Studies based on a pharmacokinetic model are marked by an asterisk in the following and in the References.

\section{Detection of prostate cancer}

When prostate cancer is suspected on clinical grounds, systematic biopsy guided by transrectal ultrasound (TRUS) is usually used to confirm the diagnosis [49]. In $66-71 \%$ of patients undergoing TRUS-guided prostate biopsy for the first time, the biopsy results are negative $[50,51]$. On the other hand, $23 \%$ of all prostate cancers detected by TRUSguided biopsy are missed in the first biopsy [51]. In other words though biopsy results are true negative in a considerable proportion of patients some patients have to undergo repeat biopsy before their cancers are detected. Initial results suggest that combining a $\mathrm{T} 2 \mathrm{w}$ sequence with phenomenological parameters derived from a DCE-MRI sequence (DCE-MRI for short), pharmacokinetic parameters, and DWI holds promise for improving cancer detection and thereby reducing the need for prostate biopsy $\left[52^{*}, 53,54 *\right]$. In patients with a prior negative TRUSguided biopsy, T2w + DCE-MRI were used to guide

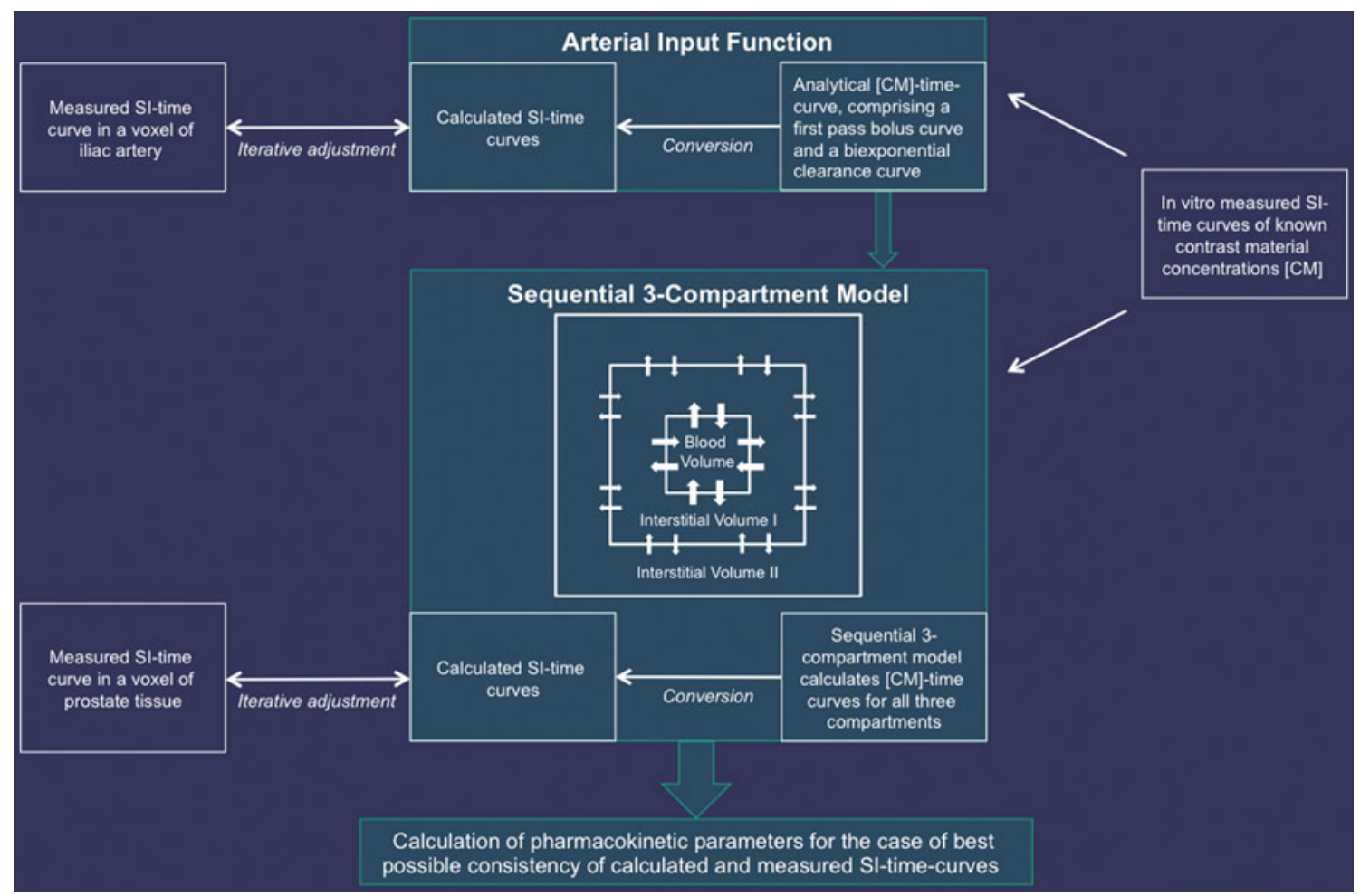

Fig. 2 Diagram for calculating pharmacokinetic parameters - blood flow, blood volume, mean transit time, permeability, extraction coefficient and interstitial volume - using the sequential 3-compartment model 
prostate repeat biopsy with a sensitivity of up to $83 \%$ and a negative predictive value of up to $100 \%$ [55]. In an exploratory study of 21 patients with more than two negative TRUS-guided biopsies and a PSA level of $>4 \mathrm{ng} / \mathrm{ml}$, targeted MRI-guided prostate biopsy detected prostate cancer in $59 \%$ of the cases after a diagnostic MRI consisting of a combination of $\mathrm{T} 2 \mathrm{w}$ images with pharmacokinetic parameters and DWI [56*] (Fig. 3). In the future, parametric maps may be fused with real-time TRUS images for targeted biopsy of suspect lesions [57].

Localization and staging of prostate cancer

Adequate treatment of prostate cancer relies on proper staging, which in turn requires accurate localization of cancer within the prostate. A study of patients scheduled for prostatectomy has shown that the surgical decision for or against preservation of the neurovascular bundles can be significantly improved by MRI staging [58]. The outcome of radiotherapy might be improved by delivering a radiation beam to the tumour identified by conventional MRI and pharmacokinetic maps [59*].

Smaller studies have already shown that conventional MRI combined with pharmacokinetic parameter maps is superior to conventional MRI alone in localizing prostate cancer $\left[60^{*}, 61^{*}\right]$ (Fig. 4). Staging accuracy in general, and detection of extracapsular extension in particular, relies on high spatial resolution, which is why imaging at $3 \mathrm{~T}$ is generally preferable to $1.5 \mathrm{~T}$ imaging, and the use of a combined endorectal/body phased-array coil is desirable $[62,63]$. The need for high spatial resolution theoretically favors the calculation of pharmacokinetic exchange parameters with the currently available MR technology, even more so as the accurate calculation of blood flow and blood volume requires high temporal resolution as well [54*]. In a study of 32 patients using a voxel volume of $1.8 \mathrm{~mm}^{3}$ for DCE-MRI, high-resolution maps of pharmacokinetic exchange parameters were significantly better than $\mathrm{T} 2 \mathrm{w}$ images for demonstrating extracapsular growth [64].

\section{Detection of recurrent prostate cancer}

Men with localized prostate cancer successfully treated by radiotherapy have cancer recurrence rates of $22-60 \%$, depending on the risk group [65]. Recurrence after prostatectomy is similar, ranging between $12 \%$ and $67 \%$ [65]. The differentiation between local recurrence and metastasis in patients with elevated PSA is difficult,
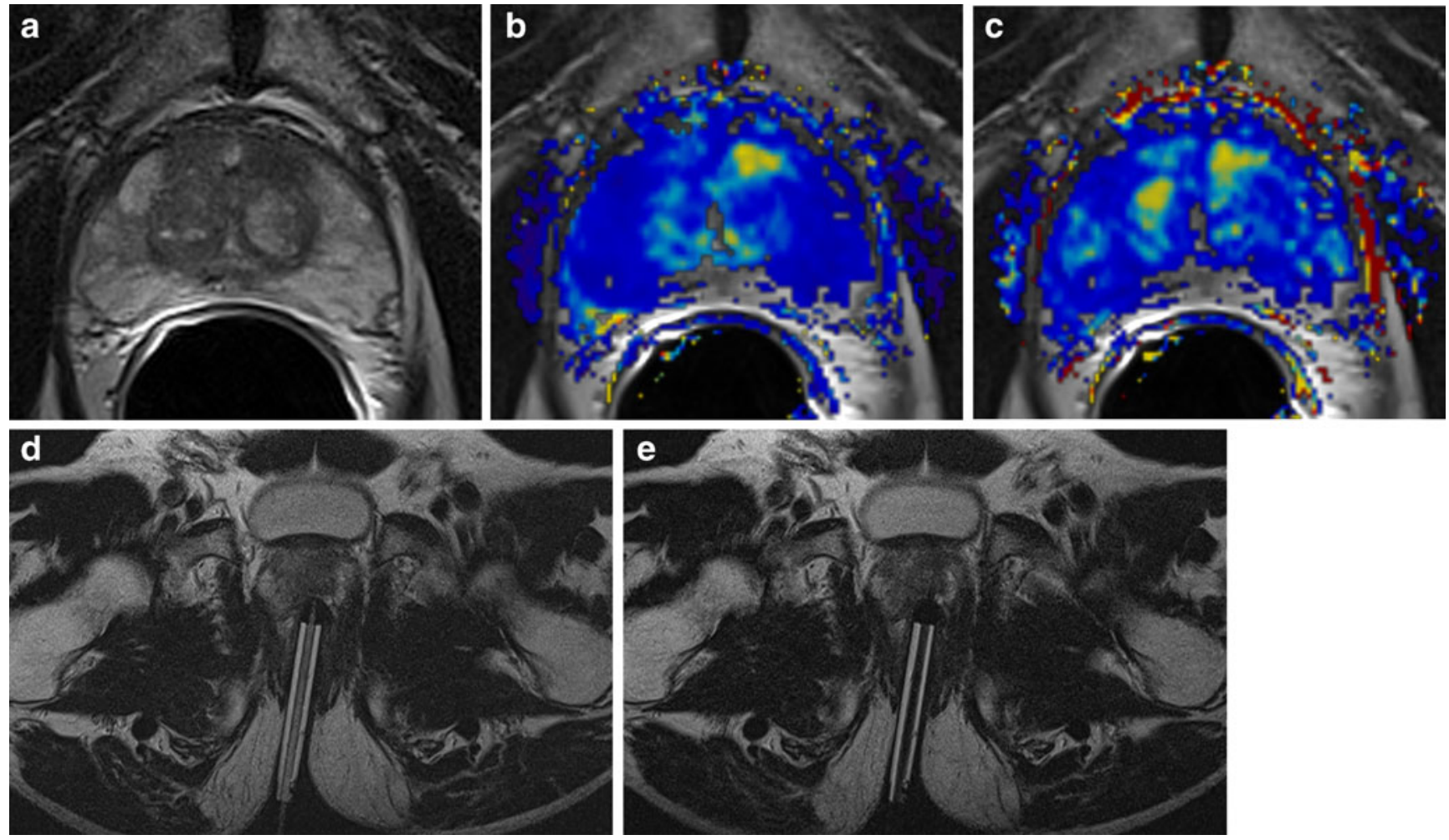

Fig. 3 a Axial T2w image of a 72-year-old patient with a current PSA level of $28.1 \mathrm{ng} / \mathrm{ml}$ and 5 prior negative TRUS-guided prostate biopsies in the last 8 years. The pharmacokinetic parameter maps for $\mathrm{K}^{\text {trans }}$ (b) and $\mathrm{k}_{\mathrm{ep}}$ (c) show an area of increased exchange constants in the left-sided anterior horn of the peripheral zone of the midgland.
This area has low signal intensity on the T2w image. MRI-guided biopsy (D+E) was performed, and prostate cancer (Gleason score, $3+$ 5) was demonstrated. Owing to the slice thickness of $4 \mathrm{~mm}$ of the $\mathrm{T} 2 \mathrm{w}$ image, the throw of the $18 \mathrm{G}$ biopsy needle is blurred in image $\mathrm{E}$ 

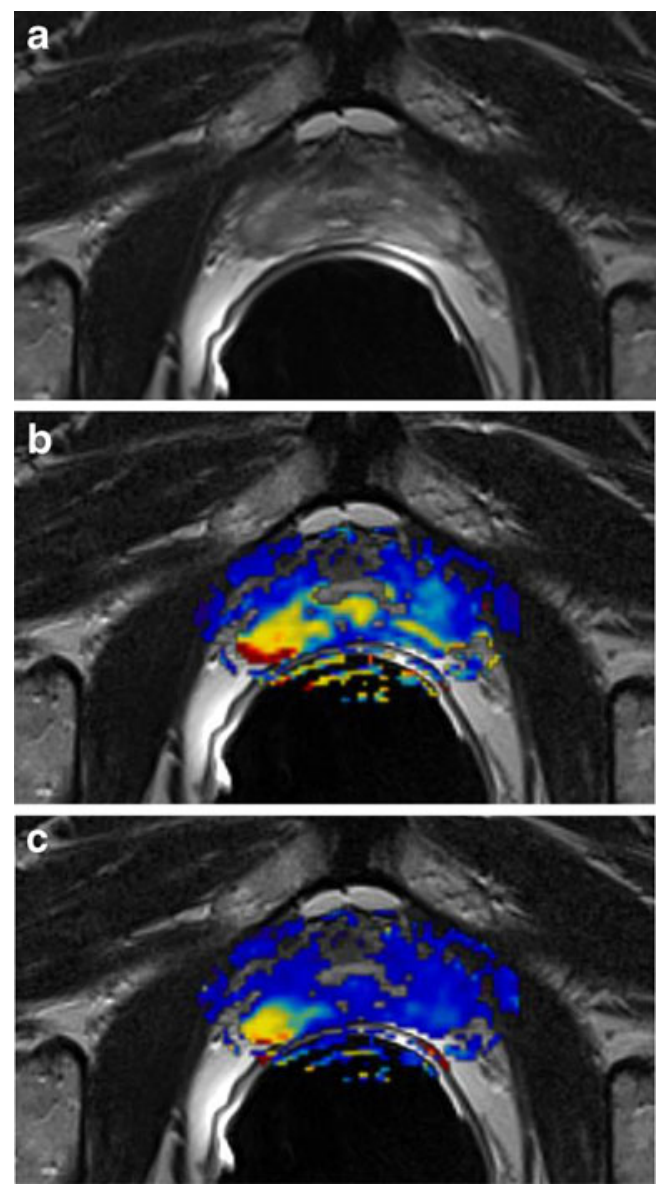

Fig. 4 a Axial T2w image of the prostate obtained in a 73-year-old patient scheduled for radical prostatectomy (PSA of $8.06 \mathrm{ng} / \mathrm{ml}$, free PSA of $0.72 \mathrm{ng} / \mathrm{ml}$ ). TRUS-guided biopsy 6 weeks earlier yielded a Gleason score of $4+3$. The $\mathrm{T} 2 \mathrm{w}$ image shows a suspicious area in the posterolateral peripheral zone near the apex on the right. The pharmacokinetic parameter maps for $\mathrm{K}^{\text {trans }}$ (b) and $\mathrm{k}_{\mathrm{ep}}$ (c) show increased values for this area. The marked increase near the capsule suggests extracapsular growth. Following prostatectomy, prostate cancer (Gleason score $4+3$ ) with a maximum transverse diameter of $13 \mathrm{~mm}$ and extracapsular extension near the right apex was confirmed. There were no other areas of cancer within the prostate

regardless of whether serum PSA [66, 67], digital rectal examination (DRE), or TRUS-guided biopsy is used to answer this question [68]. Detection of local prostate cancer recurrence is even more difficult with conventional MRI, as the low signal intensity of fibrotic changes after radiotherapy or of scar tissue after prostatectomy may mimic local recurrence on T2w images $[5,6,69,70]$.

Exploratory studies found that the additional use of DCE-MRI significantly increased sensitivity for the detection of recurrent prostate cancer after radiotherapy compared with $\mathrm{T} 2 \mathrm{w}$ images alone; the reported increase was from $38 \%$ to $72 \%$, while specificity was nearly the same, $80 \%$ versus $85 \%$ [71, 72]. Exploiting the improved accuracy of combining conventional MRI with pharmacokinetic parameter maps MR-guided biopsy detected recur- rence after radiotherapy with a positive predictive value of $68 \%[73 *]$. Furthermore, a feasibility study suggests that pharmacokinetic parameter maps are also useful for planning focal salvage treatment, which is aimed at reducing the adverse effects of radiotherapy [74*].

A similar improvement was reported for the detection of recurrence after prostatectomy as well; here the addition of DCE-MRI led to an increase in sensitivity from $61 \%$ to $84 \%$ compared with $\mathrm{T} 2 \mathrm{w}$ imaging alone, with specificity again being similar- $82 \%$ versus $89 \%$ [70]. These results were confirmed by another study [75]. It must be borne in mind, however, that the GRE sequences used for DCE-MRI may be degraded partially by severe artifacts caused by surgical clips [76].

In addition, DCE-MRI combined with T2w images might in the future also play a role in patients with suspected prostate cancer recurrence after high-intensity focused ultrasound (HIFU) treatment and for planning targeted biopsy [77].

Assessment of biological aggressiveness and estimation of prognosis

Clinical methods for the early detection of prostate cancer, particularly PSA screening, carry the risk of overdiagnosis and overtreatment [78]. The desire to avoid overtreatment has led to the introduction of active surveillance, in which definitive treatment, with its attendant risks (e.g. urinary incontinence, erectile dysfunction, cystitis, and proctitis), is delayed until progression is detected [79]. Active surveillance is heavily dependent on risk stratification based on serum PSA, Gleason score, and quantification of the proportion of cancer in biopsy specimens [80]. However, determination of these variables is not optimal and is subject to error [81-83]. Therefore, another, ideally noninvasive technique, such as imaging, would be highly desirable; its focus should be on determining the tumour aggressiveness and volume. A higher Gleason score has been shown to correlate significantly with a lower prostate cancer-to-internal obturator muscle SI ratio on conventional MR images [84]. In a study of 42 patients, low-grade prostate cancer was found to have a significantly higher blood volume and higher permeability than high-grade cancer [85*]. Studies investigating a possible correlation between MVD as a prognostic factor and individual pharmacokinetic parameters yielded inconsistent results $\left[54,85^{*}, 86^{*}, 87^{*}\right]$. The discrepancies may be attributable to the heterogeneity of vascularization in the prostate and in prostate cancer [17]. Therefore the determination of MVD in a 4- $\mu \mathrm{m}$ section may not be representative for pharmacokinetic parameters such as blood volume of a MR voxel. The usefulness of pharmacokinetic parameters as additional prognostic factors remains to be shown in studies investi- 
gating larger patient populations and over longer follow-up periods.

\section{Treatment monitoring}

Serum PSA is currently the most widely used test for monitoring the outcome of prostate cancer treatment [88]. It may take several years, though, before a PSA nadir is reached following radiotherapy $[88,89]$. During this period, nearly one third of patients will experience at least one PSA bounce of $0.3-3.4 \mathrm{ng} / \mathrm{ml}$; about half of these patients do not have recurrent cancer $[90,91]$. Such temporary increases in PSA do not necessarily indicate treatment failure, but cause great alarm to patients and physicians alike [90]. A noninvasive imaging test suitable for monitoring treatment might improve this situation by allowing earlier definitive assessment of the outcome of treatment [92]. A preliminary investigation of post-therapeutic pharmacokinetic parameters has demonstrated that blood flow and the extraction coefficient appear to be suitable for monitoring the outcome of radiotherapy and allow earlier definitive assessment than the methods currently used [93*]. Other promising approaches of therapeutic monitoring with pharmacokinetic parameters include the demonstration of a decrease in the permeability of prostate cancer tissue following hormone treatment in humans and the demonstration of a response of benign prostatic hyperplasia to pharmacotherapy in animals [94*, 95*]. Note, however, that the blood flow and permeability of tissues are altered during and after treatment $\left[93^{*}, 96^{*}\right]$. As the pharmacokinetic exchange constants, $\mathrm{K}^{\text {trans }}$ and $\mathrm{k}_{\mathrm{ep}}$, are functions of blood flow and permeability with undefined interdependencies [33], pharmacokinetic models that quantify blood flow and permeability independently of each other are preferable for treatment monitoring. Such models might also enable inter- and intraindividual comparability of data obtained by different study groups and on different MR systems [97*].

\section{Antiangiogenic therapy}

Several studies have demonstrated that inhibition of neovascularization of a malignant tumour significantly improves progression-free survival and disease-specific survival [98]. For prostate cancer, a combination of two angiogenesis inhibitors (bevacizumab and thalidomide) has been shown to inhibit the growth of metastases not responding to hormone treatment [99]. Angiogenesis inhibition might have the potential to treat localized cancer in the future, avoiding the adverse effects of the treatment options currently available. Published studies on antiangiogenic treatment in advanced prostate cancer suggest that serum PSA appears to be of limited use for assessing the response to treatment, once again underscoring the need for better diagnostic methods
[99]. With neovascularization being the histological basis for DCE-MRI, this method appears to be a good candidate for the noninvasive monitoring of treatment with angiogenesis inhibitors. This hypothesized use remains to be demonstrated in future studies investigating antiangiogenic therapy in patients with localized and advanced prostate cancer.

\section{Limitations}

At present, comparing published studies is still difficult, as investigators use different DCE-MRI sequences as well as different methods of analysis. To enable further investigation of the value of DCE-MRI of the prostate, ideally in large multicenter studies, it would desirable to arrive at a consensus on the pharmacokinetic model to be used for standardizing the method. With the currently available DCE-MRI sequences, it has not yet been possible to simultaneously achieve adequate spatial and temporal resolution in the clinical setting. This limitation might be overcome by using parallel imaging with an acceleration factor of 4-6 and with the k-t-sense approach, which interprets time as an additional dimension, making better use of the total data acquisition time for obtaining both spatial and temporal information [100]. Finally, one must not forget the risk of nephrogenic systemic fibrosis associated with the administration of gadolinium-based contrast medium, which is increased in patients with renal dysfunction.

\section{Conclusions}

To date, DCE-MRI and the corresponding pharmacokinetic models and parameters have shown great potential for improving the diagnosis of prostate cancer by adding functional information to the anatomical information provided by conventional $\mathrm{T} 2 \mathrm{w}$ and $\mathrm{T} 1 \mathrm{w}$ MR pulse sequences. An understanding of the basic physical principles underlying DCE-MRI helps radiologists to better interpret the results of DCE-MRI and of the corresponding pharmacokinetic parameters. Initial clinical applications of DCE-MRI, including the detection, localization, and staging of prostate cancer as well as the diagnosis of recurrence, demonstrate that the technique offers diagnostic benefits when compared with conventional MRI alone. Potential future applications could include estimation of a patient's prognosis and monitoring of treatment response. A definitive appraisal of the accuracy of DCE-MRI and pharmacokinetic parameters for these indications is not possible at present.

Acknowledgements This review article was written while the corresponding author, Tobias Franiel, was performing a research fellowship (supported by the German Research Foundation DFG - FR 2891/1-1) at the Memorial Sloan-Kettering Cancer Center. 


\section{References}

1. Jemal A, Siegel R, Ward E, Hao Y, Xu J, Thun MJ (2009) Cancer statistics, 2009. CA Cancer J Clin 59:225-249

2. Hricak H, Choyke PL, Eberhardt SC, Leibel SA, Scardino PT (2007) Imaging prostate cancer: a multidisciplinary perspective. Radiology 243:28-53

3. Schiebler ML, Tomaszewski JE, Bezzi M et al (1989) Prostatic carcinoma and benign prostatic hyperplasia: correlation of highresolution MR and histopathologic findings. Radiology 172:131137

4. Beyersdorff D, Taupitz M, Winkelmann B et al (2002) Patients with a history of elevated prostate-specific antigen levels and negative transrectal US-guided quadrant or sextant biopsy results: value of MR imaging. Radiology 224:701-706

5. Sala E, Eberhardt SC, Akin O et al (2006) Endorectal MR imaging before salvage prostatectomy: tumor localization and staging. Radiology 238:176-183

6. Coakley FV, Teh HS, Qayyum A et al (2004) Endorectal MR imaging and MR spectroscopic imaging for locally recurrent prostate cancer after external beam radiation therapy: preliminary experience. Radiology 233:441-448

7. Choi YJ, Kim JK, Kim N, Kim KW, Choi EK, Cho KS (2007) Functional MR imaging of prostate cancer. Radiographics 27:63-75, discussion 75-67

8. Mirowitz SA, Brown JJ, Heiken JP (1993) Evaluation of the prostate and prostatic carcinoma with gadolinium-enhanced endorectal coil MR imaging. Radiology 186:153-157

9. Brown G, Macvicar DA, Ayton V, Husband JE (1995) The role of intravenous contrast enhancement in magnetic resonance imaging of prostatic carcinoma. Clin Radiol 50:601-606

10. Engelbrecht MR, Huisman HJ, Laheij RJ et al (2003) Discrimination of prostate cancer from normal peripheral zone and central gland tissue by using dynamic contrast-enhanced MR imaging. Radiology 229:248-254

11. Nicholson B, Schaefer G, Theodorescu D (2001) Angiogenesis in prostate cancer: biology and therapeutic opportunities. Cancer Metastasis Rev 20:297-319

12. Folkman J, Cole P, Zimmerman S (1996) Tumor behavior in isolated perfused organs: in vitro growth and metastases of biopsy material in rabbit thyroid and canine intestinal segment. Ann Surg 164:491-502

13. Dewhirst MW, Tso CY, Oliver R, Gustafson CS, Secomb TW, Gross JF (1989) Morphologic and hemodynamic comparison of tumor and healing normal tissue microvasculature. Int J Radiat Oncol Biol Phys 17:91-99

14. Pallares J, Rojo F, Iriarte J, Morote J, Armadans LI, de Torres I (2006) Study of microvessel density and the expression of the angiogenic factors VEGF, bFGF and the receptors Flt-1 and FLK-1 in benign, premalignant and malignant prostate tissues. Histol Histopathol 21:857-865

15. Bigler SA, Deering RE, Brawer MK (1993) Comparison of microscopic vascularity in benign and malignant prostate tissue. Hum Pathol 24:220-226

16. Siegal JA, Yu E, Brawer MK (1995) Topography of neovascularity in human prostate carcinoma. Cancer 75:2545-2551

17. Weidner N, Carroll PR, Flax J, Blumenfeld W, Folkman J (1993) Tumor angiogenesis correlates with metastasis in invasive prostate carcinoma. Am J Pathol 143:401-409

18. Silberman MA, Partin AW, Veltri RW, Epstein JI (1997) Tumor angiogenesis correlates with progression after radical prostatectomy but not with pathologic stage in Gleason sum 5 to 7 adenocarcinoma of the prostate. Cancer 79:772-779

19. Rubin MA, Buyyounouski M, Bagiella E et al (1999) Microvessel density in prostate cancer: lack of correlation with tumor grade, pathologic stage, and clinical outcome. Urology 53:542547

20. Erbersdobler A, Isbarn H, Dix K et al (2010) Prognostic value of microvessel density in prostate cancer: a tissue microarray study. World J Urol 28:687-692

21. Concato J, Jain D, Li WW, Risch HA, Uchio EM, Wells CK (2007) Molecular markers and mortality in prostate cancer. BJU Int 100:1259-1263

22. Mucci LA, Powolny A, Giovannucci E et al (2009) Prospective study of prostate tumor angiogenesis and cancer-specific mortality in the health professionals follow-up study. J Clin Oncol 27:5627-5633

23. Huisman HJ, Engelbrecht MR, Barentsz JO (2001) Accurate estimation of pharmacokinetic contrast-enhanced dynamic MRI parameters of the prostate. J Magn Reson Imaging 13:607-614

24. Hoffmann U, Brix G, Knopp MV, Hess T, Lorenz WJ (1995) Pharmacokinetic mapping of the breast: a new method for dynamic MR mammography. Magn Reson Med 33:506-514

25. Prochnow D, Beyersdorff D, Warmuth C, Taupitz M, Gemeinhardt O, Lüdemann L (2005) Implementation of a rapid inversionprepared dual-contrast gradient echo sequence for quantitative dynamic contrast-enhanced magnetic resonance imaging of the human prostate. Magn Reson Imaging 23:983-990

26. Taillieu F, Salomon LJ, Siauve N et al (2006) Placental perfusion and permeability: simultaneous assessment with dual-echo contrast-enhanced MR imaging in mice. Radiology 241:737-745

27. de Bazelaire C, Rofsky NM, Duhamel G et al (2006) Combined $\mathrm{T} 2 *$ and $\mathrm{T} 1$ measurements for improved perfusion and permeability studies in high field using dynamic contrast enhancement. Eur Radiol 16:2083-2091

28. Walker-Samuel S, Leach MO, Collins DJ (2007) Reference tissue quantification of DCE-MRI data without a contrast agent calibration. Phys Med Biol 52:589-601

29. Pintaske J, Martirosian P, Graf H et al (2006) Relaxivity of Gadopentetate Dimeglumine (Magnevist), Gadobutrol (Gadovist), and Gadobenate Dimeglumine (MultiHance) in human blood plasma at 0.2, 1.5, and 3 Tesla. Invest Radiol 41:213-221

30. Cheng HL, Wright GA (2006) Rapid high-resolution T(1) mapping by variable flip angles: accurate and precise measurements in the presence of radiofrequency field inhomogeneity. Magn Reson Med 55:566-574

31. Port RE, Knopp MV, Brix G (2001) Dynamic contrast-enhanced MRI using Gd-DTPA: interindividual variability of the arterial input function and consequences for the assessment of kinetics in tumors. Magn Reson Med 45:1030-1038

32. Rijpkema M, Kaanders JH, Joosten FB, van der Kogel AJ, Heerschap A (2001) Method for quantitative mapping of dynamic MRI contrast agent uptake in human tumors. J Magn Reson Imaging 14:457-463

33. Tofts PS, Brix G, Buckley DL et al (1999) Estimating kinetic parameters from dynamic contrast-enhanced $\mathrm{T}(1)$-weighted MRI of a diffusable tracer: standardized quantities and symbols. J Magn Reson Imaging 10:223-232

34. Feldschuh J, Katz S (2007) The importance of correct norms in blood volume measurement. Am J Med Sci 334:41-46

35. Weinmann HJ, Laniado M, Mutzel W (1984) Pharmacokinetics of GdDTPA/dimeglumine after intravenous injection into healthy volunteers. Physiol Chem Phys Med NMR 16:167-172

36. Fritz-Hansen T, Rostrup E, Larsson HB, Sondergaard L, Ring P, Henriksen O (1996) Measurement of the arterial concentration of Gd-DTPA using MRI: a step toward quantitative perfusion imaging. Magn Reson Med 36:225-231

37. Parker GJ, Roberts C, Macdonald A et al (2006) Experimentallyderived functional form for a population-averaged hightemporal-resolution arterial input function for dynamic contrastenhanced MRI. Magn Reson Med 56:993-1000 
38. Port RE, Knopp MV, Hoffmann U, Milker-Zabel S, Brix G (1999) Multicompartment analysis of gadolinium chelate kinetics: blood-tissue exchange in mammary tumors as monitored by dynamic MR imaging. J Magn Reson Imaging 10:233-241

39. Brix G, Semmler W, Port R, Schad LR, Layer G, Lorenz WJ (1991) Pharmacokinetic parameters in CNS Gd-DTPA enhanced MR imaging. J Comput Assist Tomogr 15:621-628

40. Brix G, Schreiber W, Hoffmann U, Guckel F, Hawighorst H, Knopp MV (1997) Methodological approaches to quantitative evaluation of microcirculation in tissues with dynamic magnetic resonance tomography. Radiologe 37:470-480

41. Tofts PS, Kermode AG (1991) Measurement of the blood-brain barrier permeability and leakage space using dynamic MR imaging. 1. Fundamental concepts. Magn Reson Med 17:357367

42. Tofts PS, Berkowitz B, Schnall MD (1995) Quantitative analysis of dynamic Gd-DTPA enhancement in breast tumors using a permeability model. Magn Reson Med 33:564-568

43. Dvorak HF, Brown LF, Detmar M, Dvorak AM (1995) Vascular permeability factor/vascular endothelial growth factor, microvascular hyperpermeability, and angiogenesis. Am J Pathol 146:1029-1039

44. Gerlowski LE, Jain RK (1986) Microvascular permeability of normal and neoplastic tissues. Microvasc Res 31:288-305

45. St. Lawrence KS, Lee TY (1998) An adiabatic approximation to the tissue homogeneity model for water exchange in the brain: II. experimental validation. J Cereb Blood Flow Metab 18:1378-1385

46. Kershaw LE, Buckley DL (2006) Precision in measurements of perfusion and microvascular permeability with T1-weighted dynamic contrast-enhanced MRI. Magn Reson Med 56:986-992

47. Lüdemann L, Prochnow D, Rohlfing T et al (2009) Simultaneous quantification of perfusion and permeability in the prostate using dynamic contrast-enhanced magnetic resonance imaging with an inversion-prepared dual-contrast sequence. Ann Biomed Eng 37:749-762

48. Stephenson JL (1948) Theory of the measurement of blood flow by the dilution of an indicator. Bull Math Biophys 10:117-121

49. American Urological Association (2007) Guideline for management of clinically localized prostate cancer: 2007 update. In. http:// www.auanet.org/content/guidelines-and-quality-care/clinicalguidelines/main-reports/proscan07/content.pdf, 2007

50. Keetch DW, Catalona WJ, Smith DS (1994) Serial prostatic biopsies in men with persistently elevated serum prostate specific antigen values. J Urol 151:1571-1574

51. Roehl KA, Antenor JA, Catalona WJ (2002) Serial biopsy results in prostate cancer screening study. J Urol 167:2435-2439

52. Langer DL, van der Kwast TH, Evans AJ, Trachtenberg J, Wilson BC, Haider MA (2009) Prostate cancer detection with multi-parametric MRI: logistic regression analysis of quantitative T2, diffusion-weighted imaging, and dynamic contrast-enhanced MRI. J Magn Reson Imaging 30:327-334

53. Tanimoto A, Nakashima J, Kohno H, Shinmoto H, Kuribayashi S (2007) Prostate cancer screening: the clinical value of diffusion-weighted imaging and dynamic MR imaging in combination with T2-weighted imaging. J Magn Reson Imaging $25: 146-152$

54. * Franiel T, Lüdemann L, Rudolph B, et al (2009) Prostate MR imaging: tissue characterization with pharmacokinetic volume and blood flow parameters and correlation with histologic parameters. Radiology 252:101-108

55. Cheikh AB, Girouin N, Colombel M et al (2009) Evaluation of T2-weighted and dynamic contrast-enhanced MRI in localizing prostate cancer before repeat biopsy. Eur Radiol 19:770-778

56. * Hambrock T, Futterer JJ, Huisman HJ et al (2008) Thirty-twochannel coil 3T magnetic resonance-guided biopsies of prostate tumor suspicious regions identified on multimodality $3 \mathrm{~T}$ magnetic resonance imaging: technique and feasibility. Invest Radiol 43:686694

57. Xu S, Kruecker J, Turkbey B et al (2008) Real-time MRI-TRUS fusion for guidance of targeted prostate biopsies. Comput Aided Surg 13:255-264

58. Hricak H, Wang L, Wei DC et al (2004) The role of preoperative endorectal magnetic resonance imaging in the decision regarding whether to preserve or resect neurovascular bundles during radical retropubic prostatectomy. Cancer 100:2655-2663

59. * Jackson AS, Reinsberg SA, Sohaib SA, et al (2009) Dynamic contrast-enhanced MRI for prostate cancer localization. Br J Radiol 82:148-156

60. * Futterer JJ, Heijmink SW, Scheenen TW et al (2006) Prostate Cancer Localization with Dynamic Contrast-enhanced MR Imaging and Proton MR Spectroscopic Imaging. Radiology 241:449-458

61. * Ocak I, Bernardo M, Metzger G et al (2007) Dynamic contrastenhanced MRI of prostate cancer at 3T: a study of pharmacokinetic parameters. AJR Am J Roentgenol 189:849

62. Beyersdorff D, Taymoorian K, Knosel T et al (2005) MRI of prostate cancer at 1.5 and $3.0 \mathrm{~T}$ : comparison of image quality in tumor detection and staging. AJR Am J Roentgenol 185:1214-1220

63. Heijmink SW, Futterer JJ, Hambrock T et al (2007) Prostate cancer: body-array versus endorectal coil MR imaging at 3Tcomparison of image quality, localization, and staging performance. Radiology 244:184-195

64. Bloch BN, Furman-Haran E, Helbich TH et al (2007) Prostate cancer: accurate determination of extracapsular extension with high-spatial-resolution dynamic contrast-enhanced and T2weighted MR imaging-initial results. Radiology 245:176-185

65. D'Amico AV, Whittington R, Malkowicz SB et al (2002) Biochemical outcome after radical prostatectomy or external beam radiation therapy for patients with clinically localized prostate carcinoma in the prostate specific antigen era. Cancer 95:281-286

66. Kestin LL, Vicini FA, Ziaja EL, Stromberg JS, Frazier RC, Martinez AA (1999) Defining biochemical cure for prostate carcinoma patients treated with external beam radiation therapy. Cancer 86:1557-1566

67. Pound CR, Brawer MK, Partin AW (2001) Evaluation and treatment of men with biochemical prostate-specific antigen recurrence following definitive therapy for clinically localized prostate cancer. Rev Urol 3:72-84

68. Nudell DM, Wefer AE, Hricak H, Carroll PR (2000) Imaging for recurrent prostate cancer. Radiol Clin North Am 38:213-229

69. Sella T, Schwartz LH, Swindle PW et al (2004) Suspected local recurrence after radical prostatectomy: endorectal coil MR imaging. Radiology 231:379-385

70. Cirillo S, Petracchini M, Scotti L et al (2009) Endorectal magnetic resonance imaging at 1.5 Tesla to assess local recurrence following radical prostatectomy using T2-weighted and contrast-enhanced imaging. Eur Radiol 19:761-769

71. Haider MA, Chung P, Sweet J et al (2008) Dynamic contrastenhanced magnetic resonance imaging for localization of recurrent prostate cancer after external beam radiotherapy. Int $\mathrm{J}$ Radiat Oncol Biol Phys 70:425-430

72. Rouviere O, Valette O, Grivolat S et al (2004) Recurrent prostate cancer after external beam radiotherapy: value of contrastenhanced dynamic MRI in localizing intraprostatic tumorcorrelation with biopsy findings. Urology 63:922-927

73. * Yakar D, Hambrock T, Huisman H et al (2010) Feasibility of 3T dynamic contrast-enhanced magnetic resonance-guided biopsy in localizing local recurrence of prostate cancer after external beam radiation therapy. Invest Radiol 45:121-125

74. * Moman MR, van den Berg CA, Boeken Kruger AE et al (2010) Focal salvage guided by T2-weighted and dynamic 
contrast-enhanced magnetic resonance imaging for prostate cancer recurrences. Int J Radiat Oncol Biol Phys 76:741-746

75. Casciani E, Polettini E, Carmenini E et al (2008) Endorectal and dynamic contrast-enhanced MRI for detection of local recurrence after radical prostatectomy. AJR Am J Roentgenol 190:11871192

76. Zand KR, Reinhold C, Haider MA, Nakai A, Rohoman L, Maheshwari S (2007) Artifacts and pitfalls in MR imaging of the pelvis. J Magn Reson Imaging 26:480-497

77. Rouviere O, Girouin N, Glas L et al (2010) Prostate cancer transrectal HIFU ablation: detection of local recurrences using T2-weighted and dynamic contrast-enhanced MRI. Eur Radiol 20:48-55

78. Schroder FH, Hugosson J, Roobol MJ et al (2009) Screening and prostate-cancer mortality in a randomized European study. N Engl J Med 360:1320-1328

79. Choo R, Klotz L, Danjoux C et al (2002) Feasibility study: watchful waiting for localized low to intermediate grade prostate carcinoma with selective delayed intervention based on prostate specific antigen, histological and/or clinical progression. J Urol 167:1664-1669

80. Dall'Era MA, Cooperberg MR, Chan JM et al (2008) Active surveillance for early-stage prostate cancer: review of the current literature. Cancer 112:1650-1659

81. Coakley FV, Chen I, Qayyum A et al (2007) Validity of prostatespecific antigen as a tumour marker in men with prostate cancer managed by watchful-waiting: correlation with findings at serial endorectal magnetic resonance imaging and spectroscopic imaging. BJU Int 99:41-45

82. Kulkarni GS, Lockwood G, Evans A et al (2007) Clinical predictors of Gleason score upgrading: implications for patients considering watchful waiting, active surveillance, or brachytherapy. Cancer 109:2432-2438

83. Poulos CK, Daggy JK, Cheng L (2004) Prostate needle biopsies: multiple variables are predictive of final tumor volume in radical prostatectomy specimens. Cancer 101:527-532

84. Wang L, Mazaheri Y, Zhang J, Ishill NM, Kuroiwa K, Hricak H (2008) Assessment of biologic aggressiveness of prostate cancer: correlation of MR signal intensity with Gleason grade after radical prostatectomy. Radiology 246:168-176

85. * Franiel T, Lüdemann L, Taupitz M, Rost J, Asbach P, Beyersdorff D (2009) Pharmacokinetic MRI of the prostate: parameters for differentiating low-grade and high-grade prostate cancer. Rofo 181:536-542

86. * Schlemmer HP, Merkle J, Grobholz R et al (2004) Can preoperative contrast-enhanced dynamic MR imaging for prostate cancer predict microvessel density in prostatectomy specimens? Eur Radiol 14:309-317

87. * Kiessling F, Lichy M, Grobholz R et al (2004) Simple models improve the discrimination of prostate cancers from the peripheral gland by T1-weighted dynamic MRI. Eur Radiol 14:1793-1801
88. Roach M 3rd (2003) Commentary on a multi-institutional analysis of external beam radiotherapy for T1-T2 prostate cancer: "love the one you're with" and "do the right thing". Int J Radiat Oncol Biol Phys 57:907-909

89. Takamiya R, Weinberg V, Young CD, Sandler H, McLaughlin P, Roach M 3rd (2003) A zero PSA slope in posttreatment prostatespecific antigen supports cure of patients with long-term followup after external beam radiotherapy for localized prostate cancer. Int J Radiat Oncol Biol Phys 56:1073-1078

90. Hanlon AL, Pinover WH, Horwitz EM, Hanks GE (2001) Patterns and fate of PSA bouncing following 3D-CRT. Int $\mathrm{J}$ Radiat Oncol Biol Phys 50:845-849

91. Cavanagh W, Blasko JC, Grimm PD, Sylvester JE (2000) Transient elevation of serum prostate-specific antigen following (125)I/(103)Pd brachytherapy for localized prostate cancer. Semin Urol Oncol 18:160-165

92. Pickett B, Kurhanewicz J, Coakley F, Shinohara K, Fein B, Roach M 3rd (2004) Use of MRI and spectroscopy in evaluation of external beam radiotherapy for prostate cancer. Int J Radiat Oncol Biol Phys 60:1047-1055

93. * Franiel T, Lüdemann L, Taupitz M, Bohmer D, Beyersdorff D (2009) MRI before and after external beam intensity-modulated radiotherapy of patients with prostate cancer: the feasibility of monitoring of radiation-induced tissue changes using a dynamic contrast-enhanced inversion-prepared dual-contrast gradient echo sequence. Radiother Oncol 93:241-245

94. * Padhani AR, MacVicar AD, Gapinski CJ et al (2001) Effects of androgen deprivation on prostatic morphology and vascular permeability evaluated with MR imaging. Radiology 218:365374

95. * Jia G, Heverhagen JT, Henry H et al (2006) Pharmacokinetic parameters as a potential predictor of response to pharmacotherapy in benign prostatic hyperplasia: a preclinical trial using dynamic contrast-enhanced MRI. Magn Reson Imaging 24:721725

96. * Maxwell RJ, Wilson J, Prise VE et al (2002) Evaluation of the anti-vascular effects of combretastatin in rodent tumours by dynamic contrast enhanced MRI. NMR Biomed 15:89-98

97. * Franiel T, Lüdemann L, Rudolph B et al (2008) Evaluation of normal prostate tissue, chronic prostatitis, and prostate cancer by quantitative perfusion analysis using a dynamic contrastenhanced inversion-prepared dual-contrast gradient echo sequence. Invest Radiol 43:481-487

98. Ferrara N, Kerbel RS (2005) Angiogenesis as a therapeutic target. Nature 438:967-974

99. Kluetz PG, Figg WD, Dahut WL (2010) Angiogenesis inhibitors in the treatment of prostate cancer. Expert Opin Pharmacother $11: 233-247$

100. Tsao J, Hansen MS, Kozerke S (2006) Accelerated parallel imaging by transform coding data compression with k-t SENSE. Conf Proc IEEE Eng Med Biol Soc 1:372 